\title{
Retrospective Cost Adaptive Flow Control Using a Dielectric Barrier Discharge Actuator with Parameter-Dependent Modeling
}

\author{
Young-Chang Cho ${ }^{* 1}$, Jesse B. Hoagg ${ }^{\dagger 2}$, Dennis S. Bernstein ${ }^{\ddagger 1}$, and Wei Shyy ${ }^{\S 1}$ \\ ${ }^{I}$ Department of Aerospace Engineering, University of Michigan, Ann Arbor, MI, 48109 \\ ${ }^{2}$ Department of Mechanical Engineering, University of Kentucky, Lexington, KY, 40506
}

\begin{abstract}
Low-Reynolds number flyers with a chord Reynolds number of $10^{5}$ or below are sensitive to flow unsteadiness and require an effective flow control scheme to achieve stable flight performance. In this paper, unsteady aerodynamics under fluctuating free-stream conditions on an infinite wing with the SD7003 airfoil geometry at chord Reynolds number 1000 is controlled using a dielectric barrier discharge (DBD) actuator with the retrospective cost adaptive control algorithm. The control law, which requires knowledge of the first nonzero Markov parameter and nonminimum-phase zeros of the linearized flow-actuator model, adjusts control gains by minimizing a quadratic function of the retrospective performance. This paper extends previous research by addressing the lift stabilization mechanism under higher-magnitude disturbance conditions and assessing the variation of system-parameter estimates as functions of the impulse magnitude, free-stream velocity and nominal voltage. The sensitivity of the closed-loop performance to the accuracy of the parameter estimates is investigated by comparing the closed-loop performance with and without modeled parameter variations.
\end{abstract}

\section{Nomenclature}

$\begin{array}{ll}c & =\text { airfoil chord length } \\ C_{d} & =\text { sectional drag coefficient } \\ C_{l} & =\text { sectional lift coefficient } \\ \mathbf{E}_{\text {or }} E_{i} & =\text { electric field vector } \\ \mathbf{F}_{b} \text { or } F_{b i} & =\text { quasi-steady body force from the DBD actuator } \\ H_{i} & =i \text {-th Markov parameter } \\ n_{c} & =\text { order of the controller } \\ R e & =\text { Reynolds number } \\ U_{\infty} & =\text { free-stream speed }\left(=\sqrt{U_{x}^{2}+U_{y}^{2}+U_{z}^{2}}\right) \\ U_{I D y} & =\text { y-directional free-stream velocity for system identification } \\ t^{*} & =\text { normalized time in the flow simulation }\left(=U_{\infty} t / c\right) \\ T^{*} & =\text { normalized disturbance period }\left(=U_{\infty} T / c\right)\end{array}$

\footnotetext{
${ }^{*}$ Ph.D. Candidate, AIAA Student Member.

${ }^{\dagger}$ Assistant Professor, AIAA Member.

* Professor, AIAA Member.

${ }^{\S}$ Clarence L. "Kelly" Johnson Collegiate Professor, Department of Aerospace Engineering, University of Michigan; currently, Provost \& Chair Professor, Department of Mechanical Engineering, Hong Kong University of Science and Technology, AIAA Fellow.
} 


$\begin{array}{ll}V_{a p p} & =\text { voltage applied to the DBD actuator } \\ V_{a p p 0} & =\text { nominal voltage applied to the DBD actuator } \\ \Delta V_{a p p} & =\text { magnitude of impulse voltage } \\ x_{a c t} & =\text { distance to the } \mathrm{DBD} \text { actuator from the leading edge } \\ \alpha & =\text { angle of attack } \\ \alpha_{d} & =\text { amplitude of sinusoidal disturbance in y-directional free-stream velocity } \\ \alpha_{l} & =\text { learning rate of the adaptive controller } \\ \theta(k) & =\text { controller parameter matrix at } k \text {-th control-time step }\end{array}$

\section{Introduction}

$\mathrm{T}$ he performance of low Reynolds number flyers, specifically with Reynolds numbers less than $10^{5}$, is significantly affected by flow conditions ${ }^{1}$. Low-Reynolds-number flyers are, by necessity, lightweight and inherently vulnerable to gusty flight conditions ${ }^{2}$. Unlike higher-Reynolds-number flows, flow structures in the lowReynolds-number regime are sensitive to flow separation, laminar-turbulent transition, and flow reattachment ${ }^{3}$. Furthermore, at high angles of attack, the instability in a separated flow region induces unsteady vortex evolution, resulting in time-varying performance. Hence, low-Reynolds-number flyers require an effective flow control strategy, which accommodates various flow conditions and is responsive to flow unsteadiness.

The goals of low-Reynolds-number flow control are to remove or decrease the laminar separation bubble, promote flow reattachment through transition to turbulence, and suppress vortex structure evolutions. Among various actuation methods, the dielectric barrier discharge (DBD) actuator requires neither components for mass injection nor mechanical moving parts. Due to the simple structure of the actuator, which consists of electrodes and a thin dielectric insulator applicable to any smooth surface, the DBD actuator can be easily installed on an airfoil. The DBD actuator is known to generate a thin layer of wall jet by delivering momentum to the neutral flow field from non-thermal ionized particles using a high intensity electric field ${ }^{4}$. Because of the DBD actuator's capability for both steady and unsteady actuations up to several $\mathrm{kHz}$, the actuator can be a versatile control device. Depending on the flow and flight conditions, the duty cycle (i.e., the time duration that the actuator is turned on) as well as the amplitude of the applied voltage can be modulated to accommodate performance and power requirements.

Despite extensive efforts to accurately capture the actuator physics, a significant difference in plasma and neutral flow time scales at low Reynolds numbers ${ }^{5}$ makes numerical approaches inefficient and infeasible for most practical problems. In order to significantly decrease computational cost, instead of using high fidelity discharge models, a simplified reduced-order model representation is proposed in Ref. 6 to approximate average body force fields. This enables the simulation of complex flow fields at much lower computational cost than first principle based models. This approach has been applied successfully to flow control in low Reynolds number airfoils ${ }^{5}$ and low-pressure turbines, eliminating flow separation?

Closed-loop active flow control can be used to achieve a desired aerodynamic performance ${ }^{8}$ and eliminate the influence of disturbances. For example, in Ref. 9 a linear quadratic regulator is used to stabilize the unstable states of a reduced-order flow model constructed using the proper orthogonal decomposition technique. However, nonlinearity and high dimensionality inherent to most flow dynamics problems provoke difficulties in modeling plant dynamics and applying an appropriate control system. Moreover, dynamic characteristics of low-Reynolds number flows are significantly dependent on flow conditions, making fixed-gain control difficult. Adaptive control techniques have the advantage of tuning the feedback gains in response to the true plant dynamics and exogenous signals. Nevertheless, these techniques typically require some model information, for example, reduced-order models ${ }^{10}$ and discrete time-series models ${ }^{11}$, and identifying this model information can be challenging.

The retrospective cost adaptive control (RCAC) algorithm updates the controller parameters using the difference between the actual past control inputs and the recomputed past inputs based on the current control parameters. The control law requires knowledge of the first nonzero Markov parameter and nonminimum-phase (NMP) zeros of the flow-actuator system. The choice of performance variable and actuator location affects the existence of NMP zeros. In the previous study ${ }^{12}$, for example, it is shown that the linearized transfer function from the control to lift is nonminimum-phase with one real NMP zero, which varies according to the actuator location, Reynolds number and impulse magnitude. On the other hand, the linearized transfer function from the control to drag is minimum-phase for the test cases. 
On the other hand, flow and actuation conditions can vary the system parameters, namely, the first nonzero Markov parameter and NMP zeros. Although there are some techniques for estimating system parameters online, adaptive control with online system identification is challenging, especially considering the flow unsteadiness with relatively small time scale compared to the amount of data for the convergence of estimated parameters. If the variation of system parameters is well-defined and predictable based on measurement, the mapping from measurement to the system parameters can be incorporated with the control law, which is analogous to gainscheduling in linear control theory.

Gain-scheduling has been used for controlling nonlinear time-varying systems. In this approach linear models are constructed around a number of operating points, resulting in nonlinear gain-scheduling controllers ${ }^{13}$. For missile autopilot design $^{14}$, for example, gain-scheduling is used based on Mach number and vertical acceleration of an airair missile to static output feedback controllers. In Ref. 15, gain-scheduling is based on the mean flow profile for linear optimal control theory to laminarize turbulent channel flow. Even with the similarity in handling parametric variations for nonlinear systems, the control framework in this study only needs knowledge of system parameters and estimates of their variations, as opposed to gain-scheduling that requires explicit descriptions for control gains as well as dynamic models specific to the plant.

In this paper, the flow-control goal is to reject the influence of free-stream unsteadiness on aerodynamic lift for an infinite wing. The nonlinearity of the flow-actuator system is assessed in detail in terms of the variation of system-parameter estimates (i.e., the first nonzero Markov parameter and NMP zero estimates). Specifically, we extend the results of Ref. 12 by exploring higher-magnitude disturbances and using system-parameter estimates that vary with impulse magnitude, free-stream velocity, and control voltage. The application of variable systemparameter estimates in the control loop enhances the transient response of the flow-actuator system. The sensitivity of control performance to the choice of system-parameter estimates is explored for the flow control framework. The role of the DBD actuation in the disturbance rejection and relevant flow mechanisms are explained in detail.

\section{Aerodynamics and DBD Actuator Models}

\section{A. Fluid Dynamics Model}

The flow fields are analyzed by solving the incompressible Navier-Stokes equations using the Loci-STREAM ${ }^{16}$, a parallelized pressure-based unstructured finite volume code. Since the ion and electron states are non-equilibrium and the ion temperature is comparable to the neutral fluid, the neutral fluid is treated as being isothermal. Considering the time scale disparity between the flow and the radio frequency (RF) actuator operation, the force acting on the neutral fluid is assumed to be a quasi-steady body force. The body force felt by the neutral flow is equivalent to the Lorentz force acting on the net charge density. For the unsteady operation of the actuator only the amplitude variation of the operation voltage with time scales much larger than the RF operation is considered. In index notation, the relevant conservation equations are

$$
\begin{gathered}
\frac{\partial u_{j}}{\partial x_{j}}=0 \\
\frac{\partial u_{i}}{\partial t}+\frac{\partial\left(u_{i} u_{j}\right)}{\partial x_{j}}=\frac{F_{b i}}{\rho}-\frac{1}{\rho} \frac{\partial p}{\partial x_{i}}+v \frac{\partial}{\partial x_{j}}\left(\frac{\partial u_{i}}{\partial x_{j}}\right),
\end{gathered}
$$

where $i, j=1,2$ for 2D flow, and $F_{b i}$ is DBD body force vector defined below.

Here, $x_{i}=(x, y, z)$ is Cartesian position in the global coordinate system, $u_{i}$ is the flow velocity, $\rho$ is the density, $p$ is pressure, $v$ is the kinematic viscosity of air.

\section{B. DBD Actuator Model}

The DBD actuator model is a simplified model with linear electric field and constant net charge density $y^{5,6}$. As shown in Figure 1, this model prescribes localized body forces in a triangular plasma region bounded by two electrodes and the dielectric surface. The electric field distribution inside the plasma region is approximated by spatially linear relations

$$
\mathbf{E}^{\prime}\left(x^{\prime}, y^{\prime}, t\right)=\left(\frac{\left|\mathbf{E}^{\prime}\left(x^{\prime}, y^{\prime}, t\right)\right| k_{2}}{\sqrt{k_{1}^{2}+k_{2}^{2}}}, \frac{\left|\mathbf{E}^{\prime}\left(x^{\prime}, y^{\prime}, t\right)\right| k_{1}}{\sqrt{k_{1}^{2}+k_{2}^{2}}}\right),
$$


where

$$
\left|\mathbf{E}^{\prime}\left(x^{\prime}, y^{\prime}, t\right)\right|=E_{0}(t)-k_{1} x^{\prime}-k_{2} y^{\prime}, \quad E_{0}(t)=\frac{V_{a p p}(t)}{d},
$$

where $\left(x^{\prime}, y^{\prime}\right)$ is the actuator local coordinate system, $d$ is the insulator thickness, and $k_{1}$ and $k_{2}$ are the linearized slopes of the electric field in the $x^{\prime}$ and $y^{\prime}$ directions, respectively. This is a solution of Gauss' equation with the constant net charge density assumption. In (4), the maximum electric field intensity $E_{0}$ is defined based on the applied voltage, and the slopes $k_{1}$ and $k_{2}$ of the electric field attenuation away from the exposed electrode and dielectric surface are set to allow the breakdown voltage at the boundary with the minimum electric field strength. As shown in Ref. 5, this analytical-empirical model results in a body force component acting on the fluid, given by

$$
\mathbf{F}_{b}(x, y, t)=\rho_{c} q_{c} \delta(x, y) f_{v} \Delta t \mathbf{E}(x, y, t),
$$

where $f_{v}$ is the AC frequency of the voltage applied to the DBD actuator and $\Delta t$ is the discharge duty cycle, and $\mathbf{E}(x, y, t)$ is the electric field distribution (3) transformed to the global coordinate system. Furthermore, since the constant charge density $\rho_{c}$ with unit charge $q_{c}$ is present only inside the plasma region, $\delta(x, y)$ is set to 0 or 1 depending on the position. For this study, the active plasma region is given by the triangular region, shown in Figure 1 , where the horizontal electric field length $l_{h}=0.05 c$ and vertical length $l_{v}=0.025 c$. The discharge duty cycle is the portion of time during which effective force generation occurs in each operation cycle. Under feedback control, the applied voltage to the electrode is changed depending on the control signal, resulting in a time-varying body force. Since the reduced-order DBD model (3)-(5) is based on the quasi-steady assumption using the time scale disparity, the control input is meaningful when its timescale lies between those of low Reynolds number flow and plasma operation. The simplified DBD model has been validated against experimental data of force generation ${ }^{5}$ and maximum induced flow velocity ${ }^{17}$. A single co-flow directional DBD actuator with voltage amplitude modulation according to the control input is used in this study.

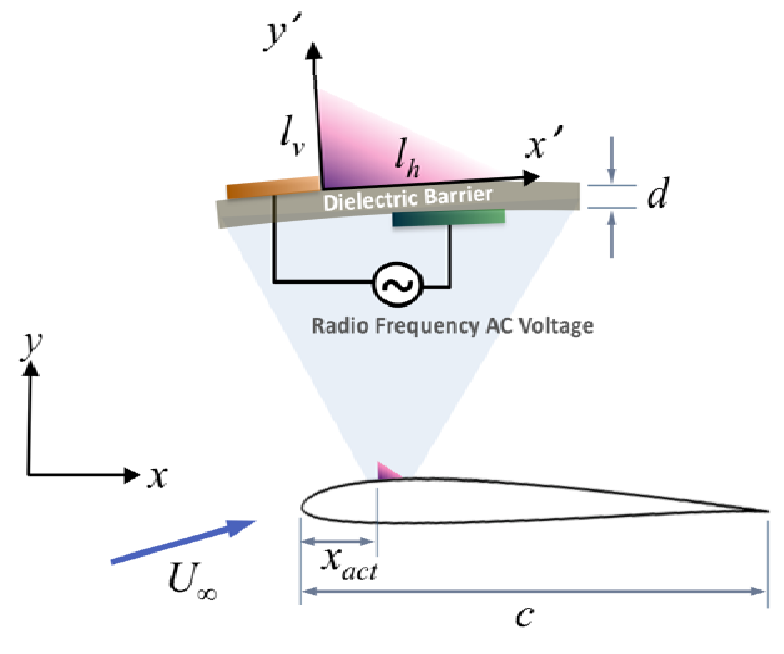

Figure 1. Schematics of the DBD actuator model.

\section{Control Algorithm}

In this section, we summarize the adaptive control algorithm presented in Ref. 18 for a single-input, singleoutput control system. The details of the multi-input, multi-output algorithm are given in Ref. 18. Consider the single-input, single-output linear discrete-time system

$$
\begin{gathered}
x(k+1)=A x(k)+B u(k)+D_{1} w(k), \\
z(k)=E_{1} x(k)+E_{0} w(k),
\end{gathered}
$$

where $x(k) \in \mathbb{R}^{n}, \mathrm{z}(k) \in \mathbb{R}, \mathrm{u}(k) \in \mathbb{R}, \mathrm{w}(k) \in \mathbb{R}^{l_{w}}$ are the state, performance, control, and exogenous command and/or disturbance signal with $k \geq 0$. We present an adaptive output feedback controller under which the performance variable $z$ is minimized in the presence of the exogenous signal $w$, which could be a disturbance, 
command, or both. In this paper, we use an adaptive feedback controller to minimize aerodynamic forces acting on the airfoil under inlet flow conditions with and without a sinusoidal disturbance.

For the general control problem given by (6)-(7), we use a strictly proper time-series controller of order $n_{c}$, such that the control $u(k)$ is given by

$$
u(k)=\sum_{i=1}^{n_{c}} M_{i}(k) u(k-i)+\sum_{i=1}^{n_{c}} N_{i}(k) y(k-i),
$$

where, for all $i=1, \cdots, n_{c}, M_{i}(k), \mathrm{N}_{i}(k) \in \mathbb{R}$ are given by the adaptive law presented below. The control can be expressed as

$$
u(k)=\theta^{T}(k) \phi(k)
$$

where

$$
\theta(k) \triangleq\left[\begin{array}{llllll}
N_{1}(k) & \cdots & N_{n_{c}}(k) & M_{1}(k) & \cdots & M_{n_{c}}(k)
\end{array}\right]^{T} \in \mathbb{R}^{2 n_{c}}
$$

is the controller parameter matrix, and the regressor vector $\phi(k)$ is given by

$$
\phi(k) \triangleq\left[\begin{array}{c}
z(k-1) \\
\vdots \\
z\left(k-n_{c}\right) \\
u(k-1) \\
\vdots \\
u\left(k-n_{c}\right)
\end{array}\right] \in \mathbb{R}^{2 n_{c}}
$$

For positive integer $\mu$, we define the extended control vector $U(k)$ by

$$
U(k) \triangleq\left[\begin{array}{c}
u(k) \\
\vdots \\
u\left(k-p_{c}+1\right)
\end{array}\right] \in \mathbb{R}^{p_{c}}
$$

where $p_{c} \triangleq \mu+1$. From (9), it follows that the extended control vector $U(k)$ can be written as

$$
U(k) \triangleq \sum_{i=1}^{p_{c}} L_{i} \theta^{T}(k-i+1) \phi(k-i+1),
$$

where

$$
L_{i} \triangleq\left[\begin{array}{c}
0_{(i-1) \times 1} \\
1 \\
0_{\left(p_{c}-i\right) \times 1}
\end{array}\right] \in \mathbb{R}^{p_{c}} .
$$

Next, define the retrospective performance

$$
\hat{z}(\hat{\theta}, k) \triangleq z(k)-\bar{B}_{z u}(U(k)-\hat{U}(\hat{\theta}, k)),
$$

where $\hat{U}(\hat{\theta}, k) \triangleq \sum_{i=1}^{p_{c}} L_{i} \hat{\theta}^{T} \phi(k-i+1), \hat{\theta} \in \mathbb{R}^{2 n_{c}}$ is an optimization variable, and control matrix $\bar{B}_{z u}$ is given by (25) below. Note that $\hat{z}(\hat{\theta}, k)$ is obtained by modifying the performance variable $z(k)$ based on the difference between the actual past control inputs $U(k)$ and the recomputed past control inputs $\hat{U}(\hat{\theta}, k)$ assuming that $\hat{\theta}$ had been used in the past. Thus, $\hat{z}(\hat{\theta}, k)$ may be interpreted as an approximation of the performance had $\hat{\theta}$ been used in the past.

Now, consider the retrospective cost function

$$
\hat{J}(\hat{\theta}, k) \triangleq \hat{z}^{2}(\hat{\theta}, k)+\alpha_{l}(\hat{\theta}-\theta(k))^{T}(\hat{\theta}-\theta(k)),
$$

where the learning rate $\alpha_{l} \in \mathbb{R}$ affects the transient performance and the convergence speed of the adaptive control algorithm. Substituting (15) into (16) yields

$$
\hat{J}(\hat{\theta}, k)=\hat{\theta}^{T} A(k) \hat{\theta}+b^{T}(k) \hat{\theta}+c(k),
$$


where

$$
\begin{aligned}
& A(k) \triangleq D^{T}(k) D(k)+\alpha_{l} I_{2 n_{c}}, \\
& b(k) \triangleq 2 D^{T}(k)\left(z(k)-\bar{B}_{z u} U(k)\right)-2 \alpha_{l} \theta(k), \\
& c(k) \triangleq\left(z(k)-\bar{B}_{z u} U(k)\right)^{2}+\alpha_{l} \theta^{T}(k) \theta(k),
\end{aligned}
$$

and $D(k) \triangleq \bar{B}_{z u} \sum_{i=1}^{p_{c}}\left(L_{i} \phi^{T}(k-i+1)\right)$. Since $A(k)$ is positive definite, $\hat{J}(\hat{\theta}, k)$ has the unique global minimizer $-\frac{1}{2} A^{-1}(k) b(k)$. Thus, the update law is given by

$$
\theta(k+1)=-\frac{1}{2} A^{-1}(k) b(k) .
$$

The adaptive controller (9) and (18)-(21) requires limited model information of the plant (6)-(7); however, the controller does require knowledge of $\bar{B}_{z u}$. We construct $\bar{B}_{z u}$ using estimates of the plant's relative degree, first nonzero Markov parameter, and the NMP zeros of the transfer function from $u$ to $z$. Consider the transfer function from $u$ to $z$ given by

which can be written as

$$
G_{z u}(z) \triangleq E_{1}(z I-A)^{-1} B
$$

$$
G_{z u}(z)=H_{d} \frac{\beta(z)}{\alpha(z)},
$$

where the relative degree $d \geq 1$ is the smallest positive integer $i$ such that the $i$-th Markov parameter $H_{i} \triangleq C A^{i-1} B$ is nonzero, and $\alpha(z)$ and $\beta(z)$ are monic coprime polynomials. Next, let $\beta(z)$ have the factorization

$$
\beta(z)=\beta_{u}(z) \beta_{s}(z),
$$

where $\beta_{s}(z)$ is a monic polynomial of degree $n_{s}$ whose roots lie inside the unit circle, and $\beta_{u}(z)$ is a monic polynomial of degree $n_{u}$ whose roots lie on or outside the unit circle. Furthermore, we can write $\beta_{u}(z)=z^{n_{u}}+\beta_{u, 1} z^{n_{u}-1}+\cdots+\beta_{u, n_{u}-1} z+\beta_{u, n_{u}}$. Then we let $\mu=n_{u}+d$ and the resulting control matrix $\bar{B}_{z u}$ is given by

$$
\bar{B}_{z u} \triangleq H_{d}\left[\begin{array}{lllll}
0_{1 \times d} & 1 & \beta_{u, 1} & \cdots & \beta_{u, n_{u}}
\end{array}\right] \in \mathbb{R}^{1 \times p_{c}} .
$$

Note that $\bar{B}_{z u}$ is constructed using knowledge of the relative degree $d$, the first nonzero Markov parameter $H_{d}$, and the NMP zeros of $\beta_{u}(z)$ of the transfer function from $u$ to $z$. Other constructions of $\bar{B}_{z u}$ are shown in Ref. 18.

The first nonzero Markov parameter and NMP zeros of the linearized flow-actuator model can be estimated by identifying Markov parameters from an impulse response. Specifically, the DBD actuator is excited by the impulse voltage $\Delta V_{a p p}$ from the nominal voltage $V_{a p p o}$. In this study, we use the difference between the instantaneous aerodynamic lift coefficient and the lift coefficient with the nominal voltage as the performance $z(k)$, and the difference between the instantaneous voltage and the nominal voltage as the control $u(k)$. As shown in the previous study $^{12}$, the linearized transfer function from the voltage to lift is a nonminimum-phase system.

Although the flow-actuator system is modeled as a linear time-invariant system, the system parameters identified in this study suggest that the system-parameter estimates vary depending on the flow conditions (i.e., geometry and flow velocity) and actuation conditions (i.e., impulse magnitude and nominal voltage). Since the flow and actuation conditions are subject to the disturbance, the linearized system model based on the system parameters identified at a specific condition can restrict the control performance as the disturbance magnitude increases. In order to reflect the variation of system-parameter estimates, $\bar{B}_{z u}$ is updated at each control-time step with the curve-fitted values, which are specified in the next section.

\section{Results and Discussion}

Flow simulations are done for the SD7003 airfoil geometry at the chord Reynolds number of 1000. Angle of attack is $15^{\circ}$ where a massive flow separation exists without actuation, and the location of the actuator $x_{a c t}$ is $0.2 c$. The DBD actuator has a nominal voltage $V_{a p p 0}$ of $2 \mathrm{kV}$, allowing the separated flow topology to exist with a reduced 
size. The time step size for flow simulation normalized by the free-stream convection time scale is 0.05 , and sampling the performance variable and updating control are done at every $10^{\text {th }}$ flow time step. A sinusoidal variation in the vertical free-stream velocity is introduced as disturbance. For each case, the performance variable is the lift coefficient of the airfoil, and the objective is to minimize the difference between the measured lift under the influence of disturbance and nominal lift without any disturbance.

\section{A. Variation of system-parameter estimates}

The system parameters - first nonzero Markov parameter and NMP zeros - are estimated from an impulse whose magnitude varies between $5 \mathrm{~V}$ and $1 \mathrm{kV}$. Since the actuator-flow system is nonlinear, the system-parameter estimates depend on the impulse magnitude. As shown in Figure 2(a), a higher impulse magnitude results in a smaller number of Markov parameters with higher magnitudes. The estimated NMP zeros shown in Figure 2(b) indicate that there exist 3 NMP zeros for the impulse magnitudes of 20 and $50 \mathrm{~V}$, whereas one real NMP zero is estimated for higher impulse magnitudes. Moreover, the real NMP zero estimate moves away from the unit circle, as the impulse magnitude increases. An impulse whose magnitude is smaller than $20 \mathrm{~V}$ hardly affects the locations of three NMP zeros.

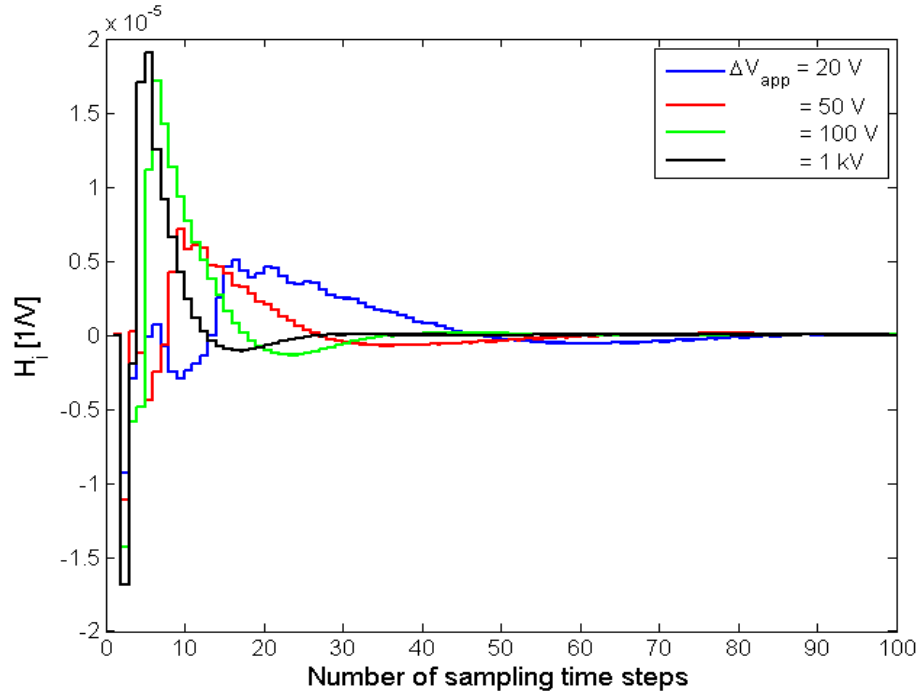

(a) Markov parameters

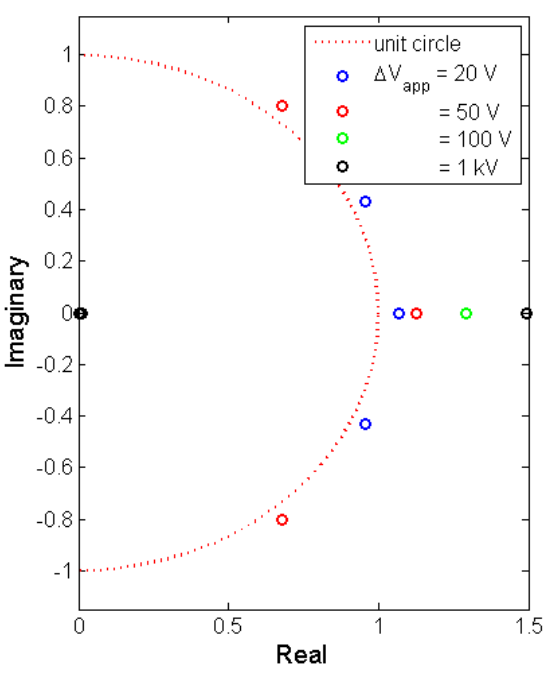

(b) estimated NMP zeros

Figure 2 System-parameter estimates depending on impulse magnitude (2D SD7003, $R e=1000, \alpha=15^{\circ}$ ).

The variation of the number and location of NMP zeros depending on the impulse magnitude results in uncertainty about determining system-parameter estimates. The closed-loop control results with different systemparameter estimates are compared in Figure 3. As shown in Figure 3(a), when three NMP zeros identified with the impulse of $\Delta V_{a p p}=5 \mathrm{~V}$ are used to constitute $\bar{B}_{z u}$, the controller, which is turned on at $t^{*}=U_{\infty} t / c=150$, fails within several disturbance cycles. If the two complex NMP zeros are excluded in constructing the system parameters, as shown in Figure 3(b), the controller fails immediately. On the other hand, when the one NMP zero identified with $\Delta V_{a p p}=200 \mathrm{~V}$ is used, the controller can suppress the lift fluctuation successfully as shown in Figure 3(c). If the impulse magnitude is increased further, for example, to $\Delta V_{a p p}=400 \mathrm{~V}$ as in Figure 3(d), the control performance deteriorates. The difficulty of stabilizing lift using 3 NMP zeros that are located near the unit circle is consistent with the well-known characteristics of nonminimum-phase systems. Furthermore, the result indicates that there exists a moderate impulse magnitude that provides a set of system-parameter estimates achieving a better control performance than others. 


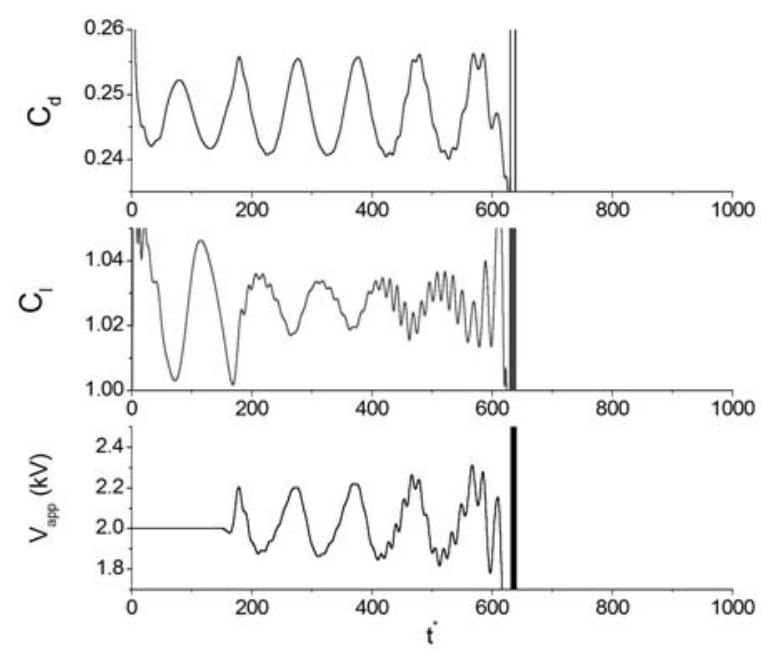

(a) 3 NMP zeros identified with $\Delta V_{a p p}=5 \mathrm{~V}$
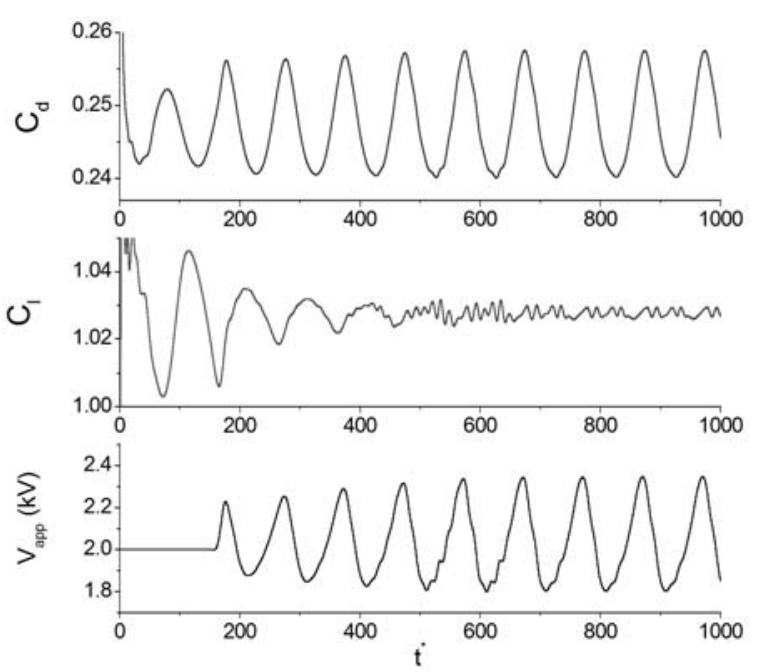

(c) 1 real NMP zero identified with $\Delta V_{a p p}=200 \mathrm{~V}$
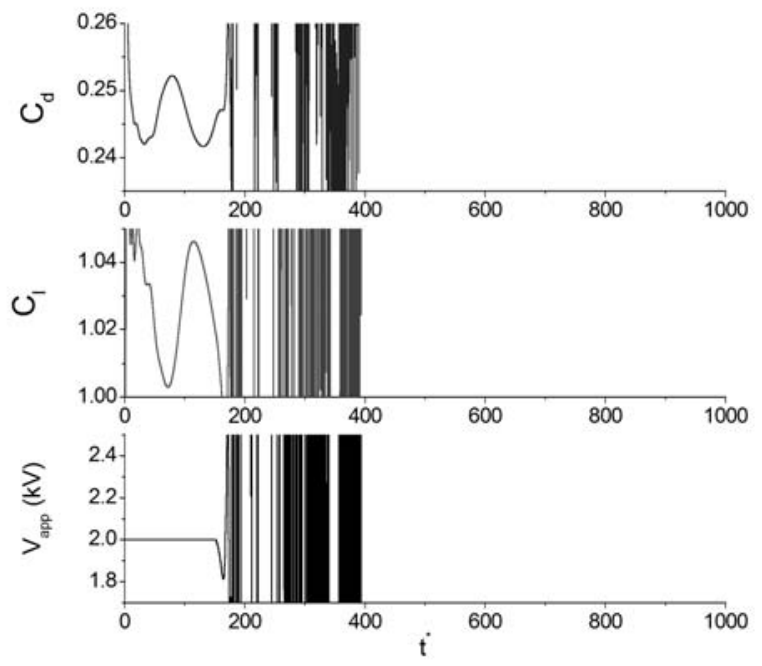

(b) 1 real NMP zero identified with $\Delta V_{a p p}=5 \mathrm{~V}$
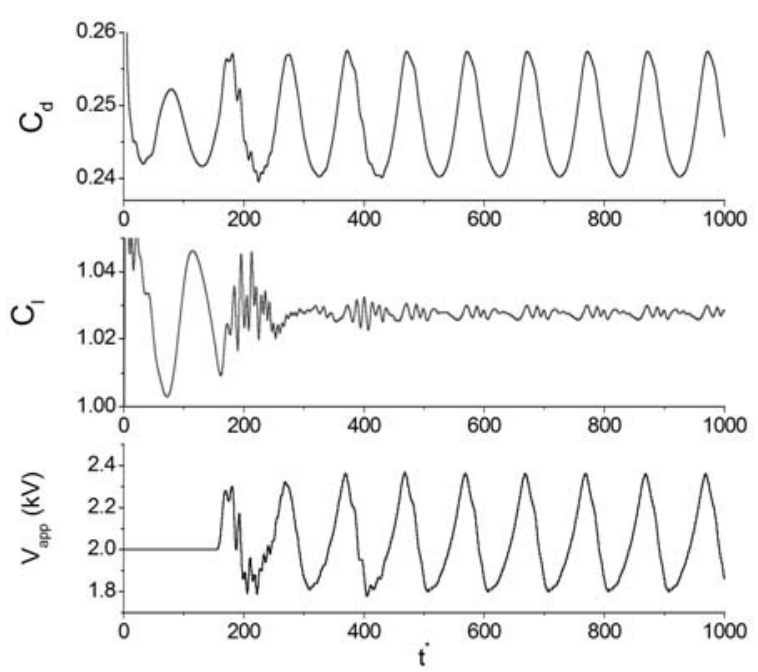

(d) 1 real NMP zero identified with $\Delta V_{a p p}=400 \mathrm{~V}$

Figure 3 Time-history of closed-loop control results with different system-parameters estimates (2D SD7003, $\left.\operatorname{Re}=1000, \alpha=15^{\circ}, x_{a c t}=0.2 c, \alpha_{d}=6 \%, T^{*}=100, \alpha_{l}=200, n_{c}=50\right)$.

The system-parameter estimates also depend on the free-stream velocity. Figure 4 shows the first nonzero Markov parameter and real NMP zero estimated by impulse response tests when the vertical free-stream velocity for system identification $U_{I D y}$ varies from the nominal vertical free-stream velocity $U_{y}$. The variation of the vertical freestream velocity changes both angle-of-attack and free-stream speed. A higher $U_{I D y}$ results in a larger angle-of-attack and the increase of free-stream speed compared to the nominal flow case. The relations between the vertical freestream velocity and system-parameter estimates can be approximated with linear curve-fits. With the $20 \%$ variation of the vertical free-stream velocity, $25 \%$ and $8 \%$ variations occur to the estimated first nonzero Markov parameter and NMP zero, respectively. Similar to the impact of the impulse magnitude on the system-parameter estimates, the variation of system parameters due to the free-stream velocity can invalidate the linear modeling of the flowactuator system when the amplitude of the free-stream disturbance increases. 

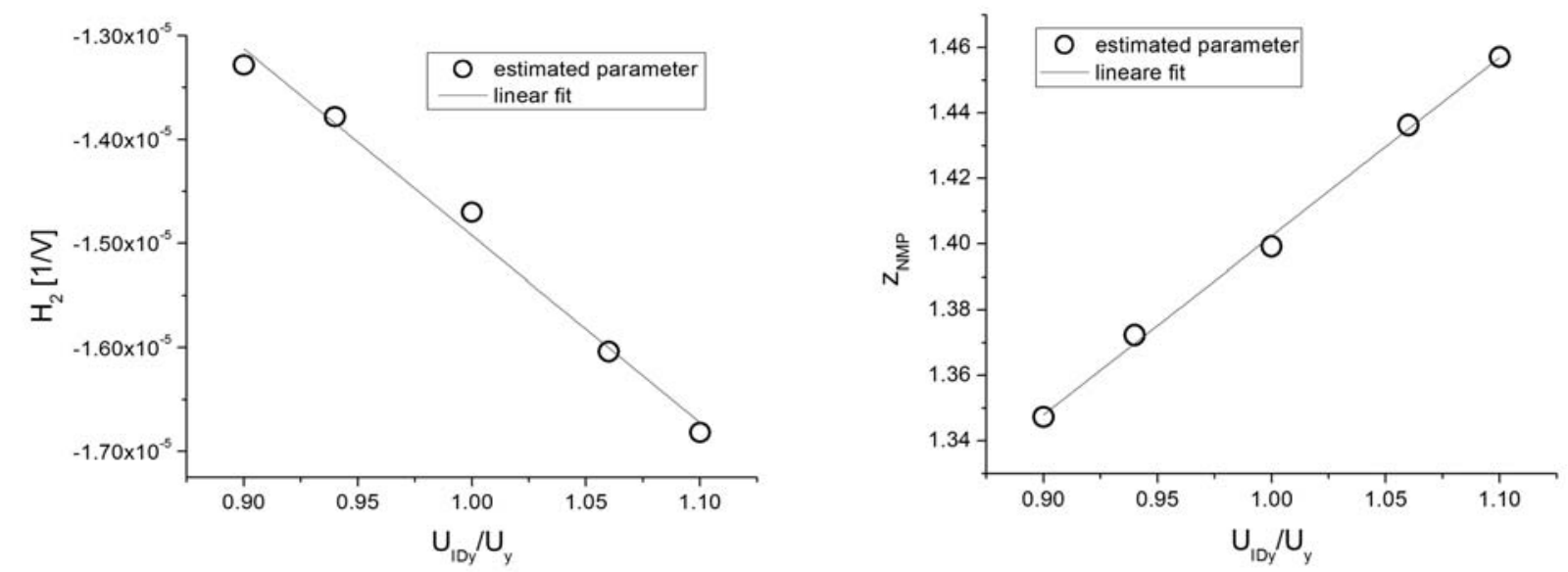

Figure 4 Variation of system-parameter estimates for lift with the vertical free-stream velocity based on 200 Markov parameters (2D SD7003, $R e=1000, \alpha=15^{\circ}, x_{a c} / c=0.2, V_{a p p 0}=2 \mathrm{kV}, \Delta V_{a p p}=0.2 \mathrm{kV}$ ).

On the other hand, the nominal voltage $V_{a p p 0}$ used for system identification can change the system-parameter estimates as shown in Figure 5. For the typical voltage range of the current study $(1 \sim 5 \mathrm{kV})$, the first nonzero Markov parameter shows about a $30 \%$ variation, whereas the real NMP zero shows a relatively small variation of $1.5 \%$. Considering that a higher nominal voltage causes the decrease of the separated flow region, it is interesting to observe the low sensitivity of the real NMP zero to the nominal voltage.
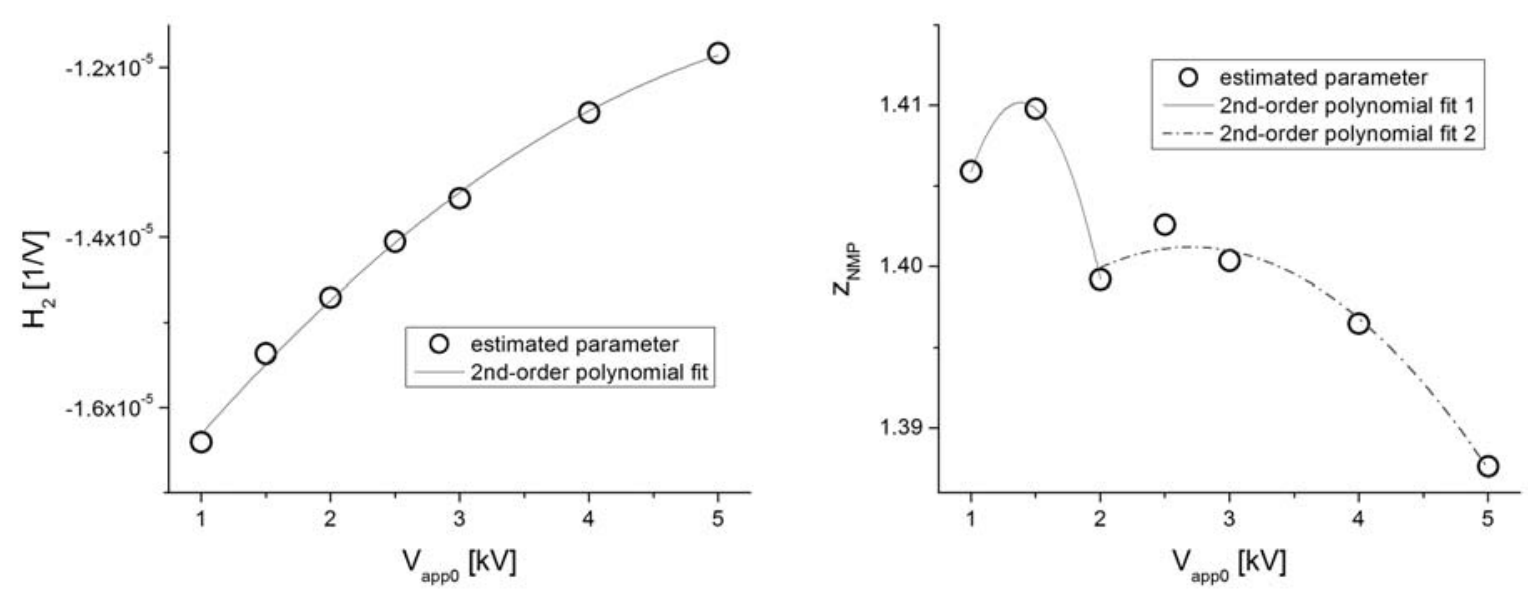

Figure 5 Variation of system-parameter estimates for lift with the nominal voltage based on 200 Markov parameters (2D SD7003, $\left.R e=1000, \alpha=15^{\circ}, x_{a c} / c=0.2, \Delta V_{a p p}=0.2 \mathrm{kV}\right)$.

The variation of the first non-zero Markov parameter due to either the change of the nominal voltage or freestream velocity can be attributed to the changes in the flow field, especially in the separated flow region. The increase of the vertical free-stream velocity results in the expansion of the separated flow region. On the other hand, the increase of the nominal voltage reduces the separated flow region by increasing the flow speed induced by the DBD actuation. In Figure 6, Markov parameters obtained from the impulse response of lift are shown for different vertical free-stream velocity and nominal voltage: the increase of the vertical free-stream results in the amplification of Markov parameters, whereas the increase of the nominal voltage results in the opposite. These trends are consistent with the variations of the first non-zero Markov parameter shown in Figure 4 and Figure 5. The results indicate that the augmented sensitivity of lift to an impulse (i.e., the amplification of Markov parameters) with the increase of the vertical free-stream or decrease of the nominal voltage, are related to the expansion of the separated flow region.

In order to include the variations of system-parameters, as shown in Figure 4 and Figure 5, the impacts of the vertical free-stream velocity and the nominal voltage on the system-parameter estimates are curve-fitted using first- 
and second-order polynomials, respectively. The closed-loop control performance with the variable systemparameter estimates is discussed in section D.

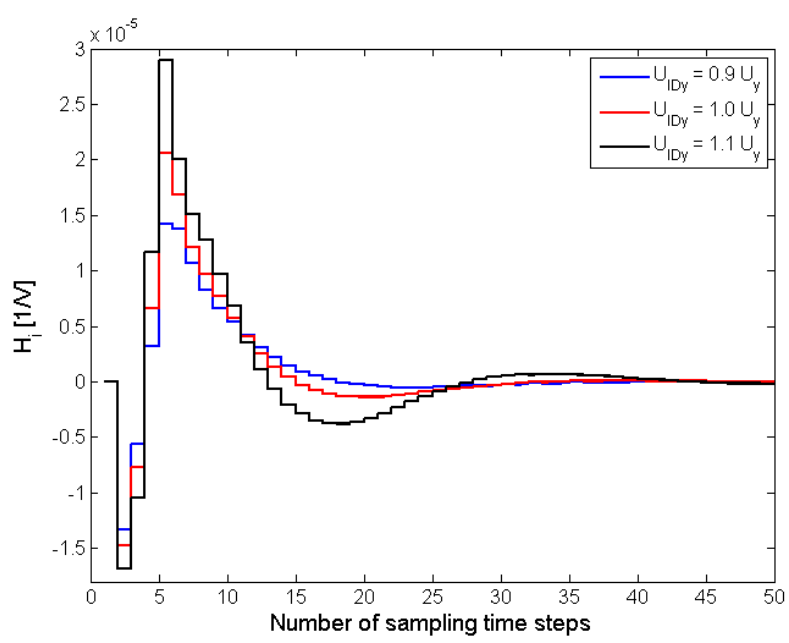

(a) $V_{\text {app } 0}=2 \mathrm{kV}$

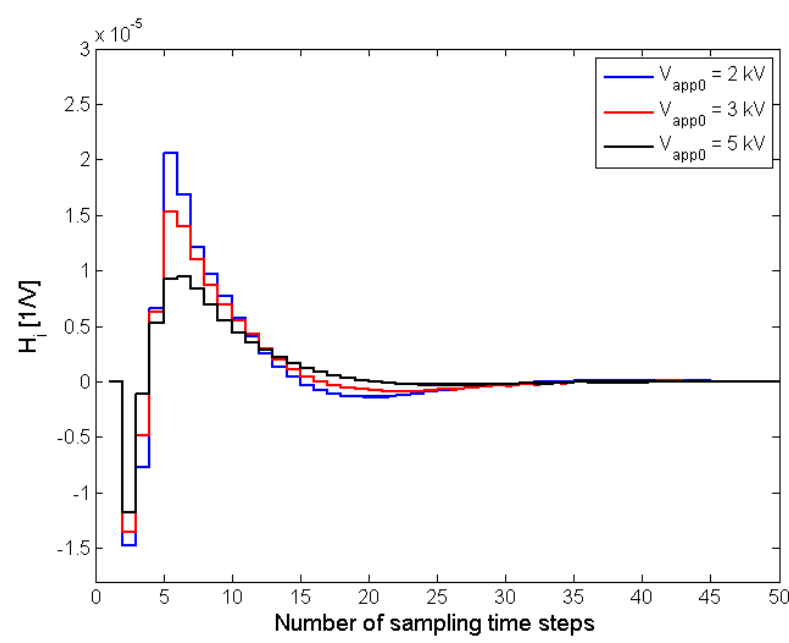

(b) $U_{I D y}=1.0 U_{y}$

Figure 6 Markov parameters depending on vertical free-stream velocity and nominal voltage

\section{B. Closed-loop control with fixed system-parameter estimates}

The previous study ${ }^{12}$ shows that the control system fails with the disturbance amplitude $\alpha_{d} \geq 15 \%$. The failure starts in the beginning of the transient response in the form of severe fluctuations of the control voltage. For the disturbances with a lower disturbance frequency, for example, $T^{*}=U_{\infty} T / c=300$, the control failure can be prevented by increasing the learning rate $\alpha_{l}$. As shown in Figure 7, even with some initial fluctuation, for $\alpha_{l}=1200$ (compared to $\alpha_{l}=200$ in Figure 3), the control voltage is reasonably bounded and can stabilize the lift fluctuation within several disturbance cycles.

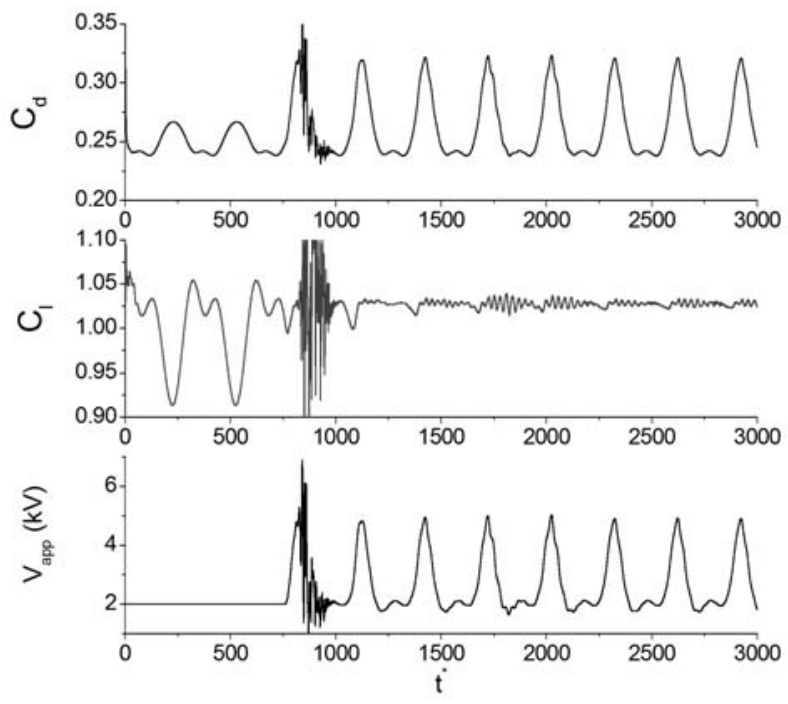

Figure 7 Time history of aerodynamic forces and control (2D SD7003, $R e=1000, \alpha=15^{\circ}, x_{a c t}=0.2 c, \alpha_{d}=20$

$$
\%, T^{*}=300, \alpha_{l}=1200, n_{c}=50,1 \text { NMP zero identified with } \Delta V_{a p p}=0.2 \mathrm{kV} \text { ). }
$$

For disturbances with lower $\alpha_{d}$, (for example, $\alpha_{d}=6 \%$ in Figure 3), the lift fluctuation is almost symmetric with respect to the undisturbed lift. The lift-stabilization mechanism of the DBD actuation shown in Ref. 12 can be summarized as the pressure variation in the pressure and suction regions. In order to neutralize the lift variation due to the disturbance, the controller decreases or increases the applied voltage according to the increase or decrease of the vertical free-stream velocity, respectively. For a higher $\alpha_{d}$ of $20 \%$, on the other hand, the lift fluctuation is biased toward the lower values compared to the target lift (i.e., the undisturbed lift coefficient of 1.03) as shown in 
Figure 7, in addition to the increased fluctuation amplitude. Figure 8 shows time evolutions of aerodynamic forces and control voltage for one-disturbance cycle enlarged from Figure 7 at two temporal windows. In Figure 8(a), for the open-loop actuation with a constant voltage, the first-half disturbance cycle (between instants 1 and 6) shows a slight fluctuation of lift around the undisturbed lift coefficient. The major impact of the disturbance on lift occurs in the second-half cycle in the form of a dip. Consequently, the corresponding control effort is mainly the increase of voltage in the second half-cycle of the disturbance, as shown in Figure 8(b).

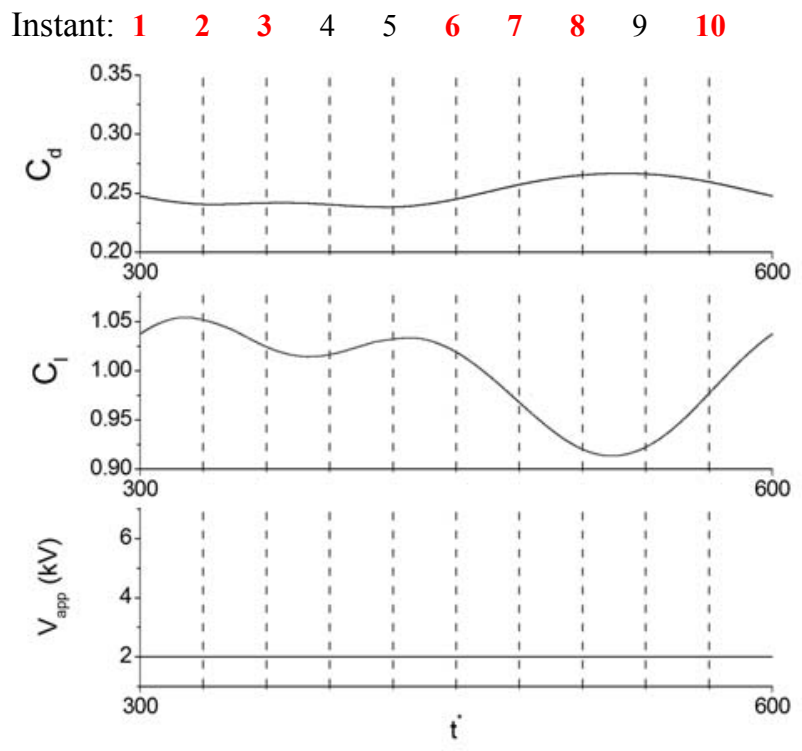

(a) open-loop actuation

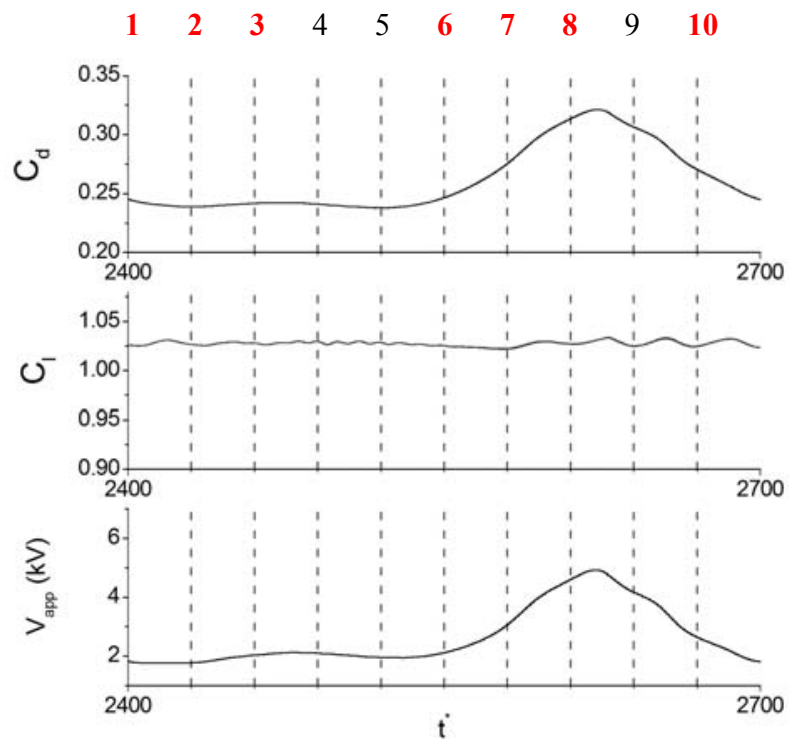

(b) closed-loop actuation

Figure 8 Time history of aerodynamic forces and control for one disturbance period from Figure 7.
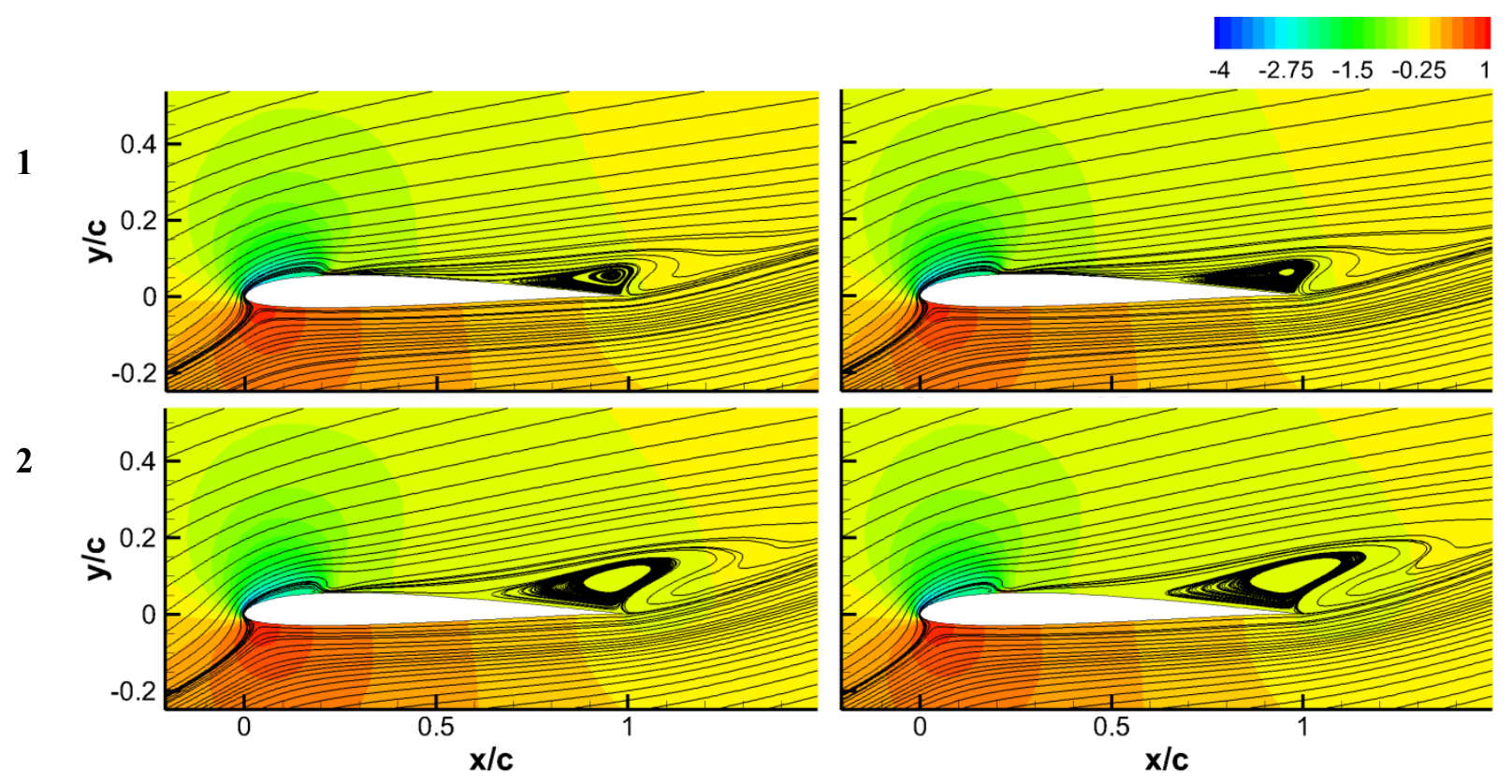


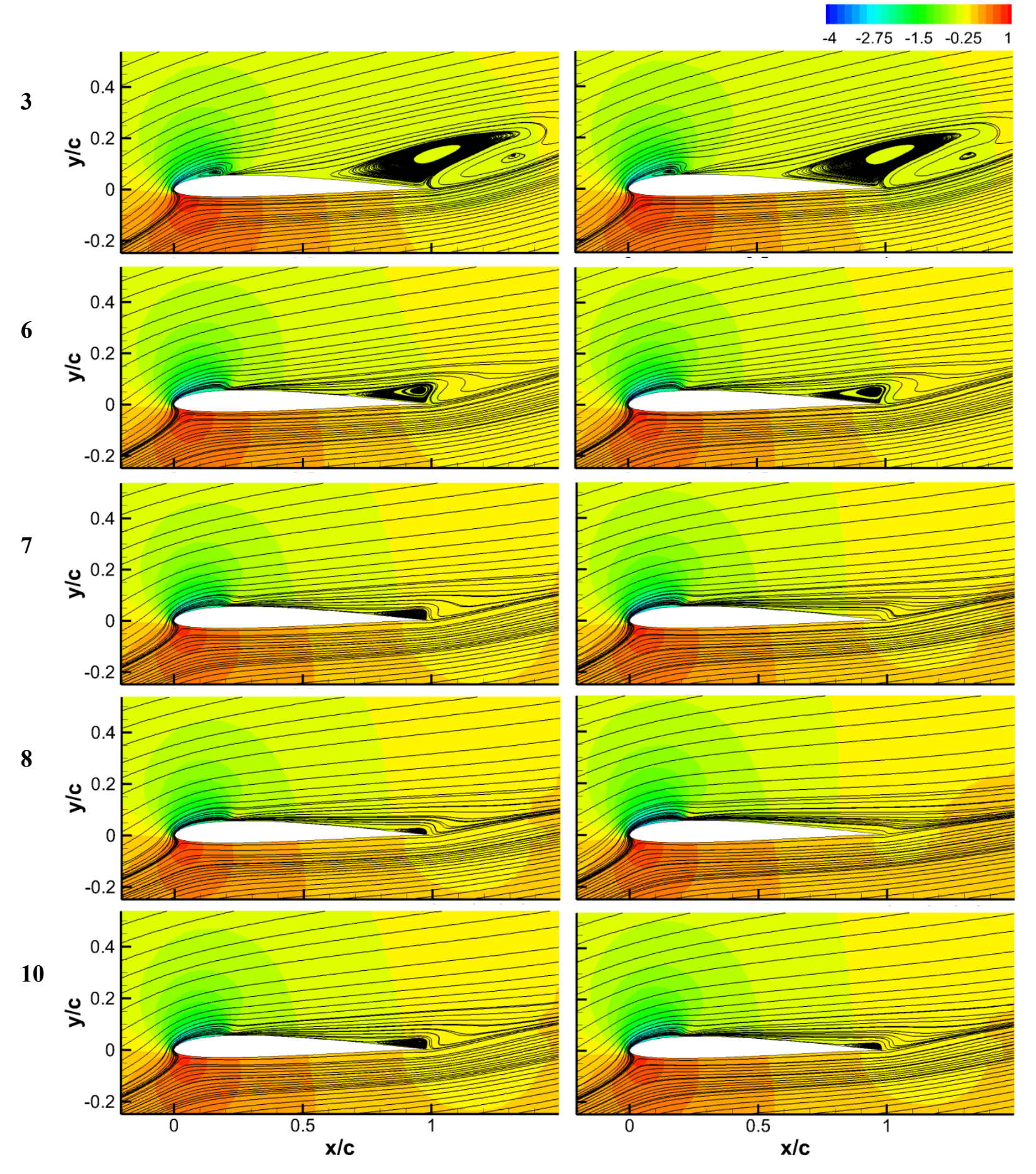

Figure 9 Pressure coefficient $\left(C_{p}\right)$ contours and streamlines corresponding to the time instants in Figure 8; left column: open-loop actuation, right column: closed-loop actuation.

The 7 instants out of 10 evenly distributed time instants in Figure 8 are chosen, and the corresponding flow-field snapshots are compared in Figure 9: open-loop actuation in the left column and closed-loop actuation in the right column. As shown in Figure 8(b), for the closed-loop actuation, the control voltage initially decreases slightly below the nominal voltage of $2 \mathrm{kV}$, resulting in a slight increase of the separated flow region at instants 1 and 2 as shown in Figure 9. For the second half-cycle, the controller increases voltage to compensate for the lift dip due to the disturbance, resulting in the intensified suction region near the actuator and the decrease or removal of the separated 
flow region at instants 6 through 10. The pressure contours show that the modification of the separated flow region in the second-half cycle exerts a minor impact on the lift stabilization compared to the change in the suction region, which is reasonable considering the low Reynolds number of 1000 .

\section{Impact of disturbance frequency}

The disturbance frequency affects the closed-loop control performance, especially the amplitudes of transient and steady-state fluctuations. For example, two disturbance-rejection cases with different disturbance periods are compared in Figure 10 while keeping the other parameters same. Both results are shown in the time scale normalized by the disturbance period. As shown in Figure 10(a), the lift and drag variations of the two cases with the constant $2 \mathrm{kV}$ actuation are similar except the peak lift values. After the closed-loop control starts at $t^{*} / T^{*}=2.5$, the control voltage accompanies divergent oscillations in the transient response, which is more severe for $T^{*}=100$. Although the amplitude of the lift fluctuation is reduced in the steady state, the closed-loop control results in significant high-frequency oscillations. On the other hand, for the lower frequency of $T^{*}=300$, the transient oscillation is more bounded, and the lift fluctuation is stabilized more efficiently. The enlarged time evolutions in Figure 10(b) shows higher lift fluctuations, especially when $V_{a p p}$ is close to $2 \mathrm{kV}$, for $T^{*}=100$. The drag variation, on the other hand, is proportional to the voltage increase for both disturbance frequencies.

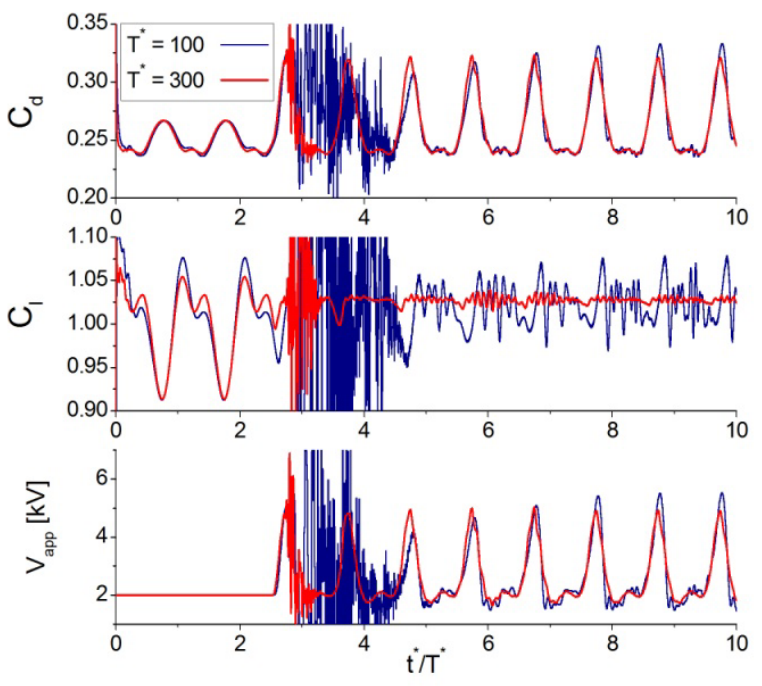

(a) 10 disturbance cycles

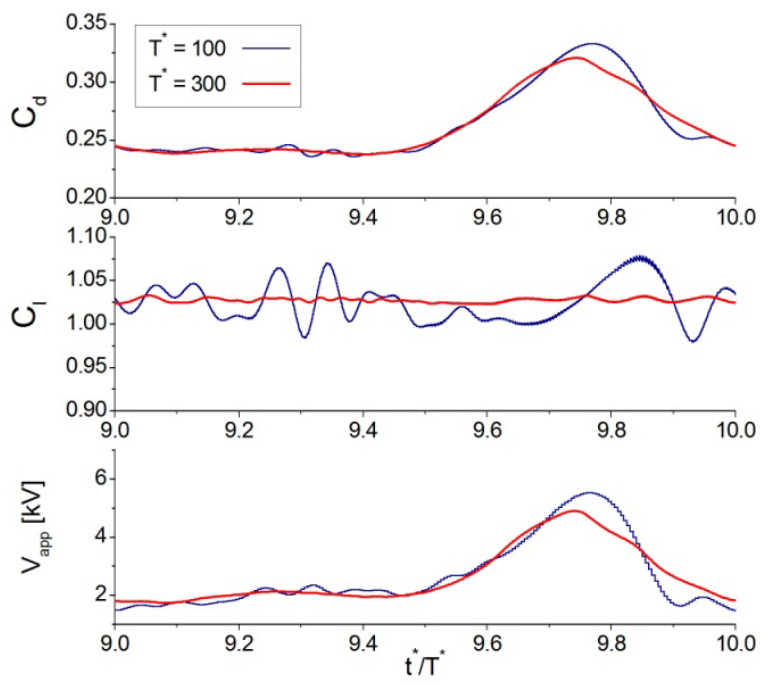

(b) 1 cycle of (a)

Figure 10 Comparison of closed-loop control results with different disturbance frequency; lift stabilization (2D SD7003, $R e=1000, \alpha=15^{\circ}, x_{a c} / c=0.2, \alpha_{d}=20 \%, \alpha_{l}=1200, n_{c}=50$ ).

More exercises suggest that the transient oscillation can be reduced by increasing the learning rate $\alpha_{l}$. For example, by increasing $\alpha_{l}$, the transient response of $T^{*}=100$ in Figure 10 can be enhanced as shown in Figure 11 in the next section. However, a higher $\alpha_{\text {l, }}$, i.e., a slower learning rate, can result in a worse steady-state result. Especially for the case with a higher-frequency disturbance, a slower learning rate limits the variation of controller parameters in response to the disturbance. As a result, the increase of $\alpha_{l}$, which can prevent the transient oscillation, can deteriorate the steady-state response for a high-frequency disturbance.

\section{Closed-loop control with variable system-parameter estimates}

The variation of system-parameter estimates is incorporated with the adaptive controller by updating the system matrix $\bar{B}_{z u}$ according to the vertical free-stream velocity and actuation voltage. Updating the first nonzero Markov parameter and real NMP zero as functions of free-stream velocity results in an insigngificant impact on either the transient or steady-state response. On the other hand, for the system-parameter estimates as functions of the actuation voltage, the case with variable system-parameter estimates is compared with the fixed system-parameter case as shown in Figure 11. For the variable system-parameter case, although there exist fluctuations with higher amplitude than the fixed system-parameter case, the transient lift fluctuations can be alleviated. The steady-state response is slightly improved or comparable to the fixed system-parameter case. The time instants with high lift fluctuations correspond to the instants when the controller parameters, $M_{I}(k)$ and $N_{l}(k)$, for example, show sudden 
changes, for example, $t^{*}=500 \sim 600$ for the fixed system-parameter and $t^{*}=800$ for the variable system-parameter case. The undesirable steady-state lift fluctuation is considered as the result of the interplay between variations of system parameters and controller parameters. However, further study is necessary to elucidate the interaction in addition to the effect of the learning rate.

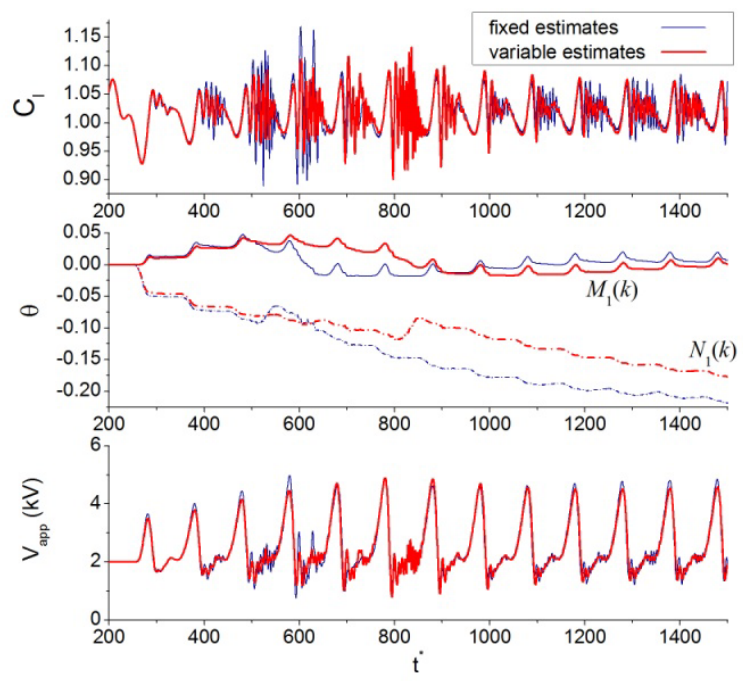

Figure 11 Comparison of closed-loop control results with fixed and variable system-parameter estimates; lift stabilization (2D SD7003, $R e=1000, \alpha=15^{\circ}, x_{a c} / c=0.2, T^{*}=100, \alpha_{d}=20 \%, \alpha_{l}=12000, n_{c}=50$ ).

\section{Summary and Conclusions}

In this study, numerical simulations of flow control using the DBD actuator are done for the SD7003 airfoil geometry at the chord Reynolds number of 1000. The control goal is to stabilize the airfoil lift under the influence of the sinusoidal disturbance in the incoming flow velocity. The closed-loop control of the flow-actuator system is achieved with the RCAC algorithm, which requires knowledge of first nonzero Markov parameter and NMP zeros as system parameters.

While identifying the system parameters impulse responses, the variation of system-parameter estimates is assessed as functions of the impulse magnitude, free-stream velocity, and nominal voltage. Considering the parametric variation as the major nonlinearity in the linearized flow-actuator system, it is shown that the choice of the system parameters affects the control performance significantly, and the impact of including the variation of system-parameter estimates in the adaptive controller is addressed.

As an extension to the previous work, which involves relatively lower disturbances with the amplitude less than $10 \%$ of the vertical free-stream velocity, stabilizing lift fluctuation under higher $(20 \%$ of the vertical free-stream velocity) disturbance amplitudes is attempted. Furthermore, the lift stabilization mechanisms of low- and highdisturbance conditions are compared. At the Reynolds number of 1000, the pressure change near the DBD actuator is the principal source of compensating for the lift changes for both low- and high-disturbance conditions. In addition, the major actuation effort occurs in the second half-cycle for the high-disturbance condition.

Comparing the control results with different disturbance frequencies, the control performance in both transient and steady-state responses can be deteriorated as the disturbance frequency increases. A higher learning rate, which reduces the convergence speed of the control gains, can enhance the transient response, but worsen the steady-state response. It is considered that as the disturbance frequency increases, the convergence of controller parameters becomes more difficult due to the faster variation of system parameters.

When the variable system-parameter estimates as functions of the applied voltage are incorporated with the adaptive controller, the transient oscillation can be enhanced. However, further study is necessary to include the variation of system parameters in the system matrix more effectively.

\section{References}

${ }^{1}$ Mueller, T. J., and DeLaurier, J. D., "Aerodynamics of small vehicles," Ann. Rev. Fluid Mech., Vol. 35, 2003, pp. 89-111. 
${ }^{2}$ Shyy, W., Lian, Y., Tang, J., Viieru, D., and Liu, H., Aerodynamics of Low Reynolds Number Flyers, New York, Cambridge Univ. Press, 2008

${ }^{3}$ Lissaman, P. B. S., "Low-Reynolds-number airfoils," Ann. Rev. Fluid Mech., Vol. 15, 1983, pp. 223-239.

${ }^{4}$ Moreau, E., "Airflow control by non-thermal plasma actuators," J. Phys. D: Appl. Phys., Vol. 40, 2007, pp. 605-636.

${ }^{5}$ Cho, Y.-C., Fledderjohn, M., Holzel, M., Jayaraman, B., Santillo, M., Bernstein, D. S., and Shyy, W., "Aadptive flow control of low Reynolds number aerodynamics using a dielectric barrier discharge actuator," 47th AIAA Aerospace Sciences Meeting Including The New Horizons Forum and Aerospace Exposition, Orlando, FL, 5-8 January, 2009, AIAA 2009-378.

${ }^{6}$ Shyy, W., Jayaraman, B., and Andersson, A., "Modeling of glow discharge-induced fluid dynamics," J. Appl. Phys., Vol. 92, No. 11, 2002, pp. 6434-6443.

${ }^{7}$ Visbal, M. R., Gaitonde, D. V., and Roy, S., "Control of transitional and turbulent flows using plasma-based actuators," $36 t h$ AIAA Fluid Dynamics Conference and Exhibit, San Francisco, CA, 5-8 June, 2006, AIAA 2006-3230.

${ }^{8}$ Taira, K., and Colonius, T., "Effect of tip vortices in low-Reynolds-number poststall flow control," AIAA Journal, Vol. 47, No. 3, 2009, pp. 749-756.

${ }^{9}$ Ahuja, S., and Rowley, C. W., "Low-dimensional models for feedback stabilization of unstable steady states," 46th AIAA Aerospace Sciences Meeting and Exhibit, Reno, NV, 7-10 January, 2008, AIAA 2008-553.

${ }^{10}$ Muse, J. A., Tchieu, A. A., Kutay, A. T., Chandramohan, R., Calise, A. J., and Leonard, A., "Vortex model based adaptive flight control using synthetic jets," AIAA Guidance, Navigation, and Control Conference, Chicago, IL, 10-13 August, 2009, AIAA 2009-5761.

${ }^{11}$ Fledderjohn, M., Cho, Y.-C., Hoagg, J. B., Santillo, M., Shyy, W., and Bernstein, D. S., "Retrospective cost adaptive flow control using a dielectric barrier discharge actuator," AIAA Guidance, Navigation, and Control Conference, Chicago, Illinois, 1013 August, 2009, AIAA 2009-5857.

${ }^{12}$ Cho, Y.-C., Hoagg, J. B., Bernstein, D. S., and Shyy, W., "Retrospective cost adaptive control of low-Reynolds number aerodynamics using a dielectric barrier discharge actuator," 40th Fluid Dynamics Conference and Exhibit, Chicago, IL, 28 June-1 July, 2010, AIAA 2010-4841.

${ }^{13}$ Rugh, W. J., and Shamma, J. S., "Research on gain scheduling," Automatica, Vol. 36, 2000, pp. 1401-1425.

${ }^{14}$ Theodoulis, S., and Duc, G., "Missile autopilot design: gain-scheduling and the gap metric," Journal of Guidance, Control, and Dynamics, Vol. 32, No. 3, 2009, pp. 986-996.

${ }^{15}$ Hogberg, M., Bewley, T. R., and Henningson, D. S., "Relaminarization of $\mathrm{Re}_{\tau}=100$ turbulence using gain scheduling and linear state-feedback control," Physics of Fluids, Vol. 15, No. 11, 2003, pp. 3572-3575.

${ }^{16}$ Kamakoti, R., Thakur, S., Wright, J., and Shyy, W., "Validation of a new parallel all-speed CFD code in a rule-based framework for multidisciplinary applications," 36th AIAA Fluid Dynamics Conference and Exhibit, San Francisco, CA, 5-8 June, 2006, AIAA 2006-3063.

${ }^{17}$ Grundmann, S., Klumpp, S., and Tropea, C. "Experimental and numerical investigations of boundary-layer influence using plasma-actuators," Active Flow Control, NNFM 95. Springer, New York, 2007, pp. 56-68.

${ }^{18}$ Santillo, M. A., and Bernstein, D. S., "Adaptive control based on retrospective cost optimization," Journal of Guidance, Control, and Dynamics, Vol. 33, No. 2, 2010, pp. 289-304. 